\title{
Gas Transport across Carbon Nitride Nanopores: A Comparison of van der Waals Functionals against the Random-Phase Approximation
}

\author{
Mohammad Tohidi Vahdat, Davide Campi, Nicola Colonna, Nicola Marzari,* \\ and Kumar Varoon Agrawal*
}

Cite This: J. Phys. Chem. C 2021, 125, 18896-18904

Read Online

ACCESS | Lلll Metrics \& More | 国 Article Recommendations | s supporting Information

ABSTRACT: $\mathrm{C}_{2} \mathrm{~N}$ is an ordered two-dimensional carbon nitride with a high density $\left(1.7 \times 10^{14} \mathrm{~cm}^{-2}\right)$ of $3.1 \AA$-sized nanopores, making it promising for highflux gas sieving for energy-efficient $\mathrm{He}$ and $\mathrm{H}_{2}$ purification. Herein, we discuss the accurate calculation of potential energy surfaces for $\mathrm{He}, \mathrm{H}_{2}, \mathrm{~N}_{2}$, and $\mathrm{CO}_{2}$ across $\mathrm{C}_{2} \mathrm{~N}$ nanopores, to characterize the gas-sieving potential of $\mathrm{C}_{2} \mathrm{~N}$. We compare the potential energy surface derived from density-functional theory calculations using five commonly used van der Waals (vdW) approximations. While all five functionals point that the $\mathrm{C}_{2} \mathrm{~N}$ nanopore yields $\mathrm{He} / \mathrm{N}_{2}$ and $\mathrm{H}_{2} / \mathrm{N}_{2}$ selectivities over 1000, adsorption energies and energy barriers vary remarkably depending on the approximation chosen. To make progress, we compare the calculations against the results from the adiabatic connection fluctuation dissipation theory, with random-phase approximation, known to be accurate in capturing vdW interactions.

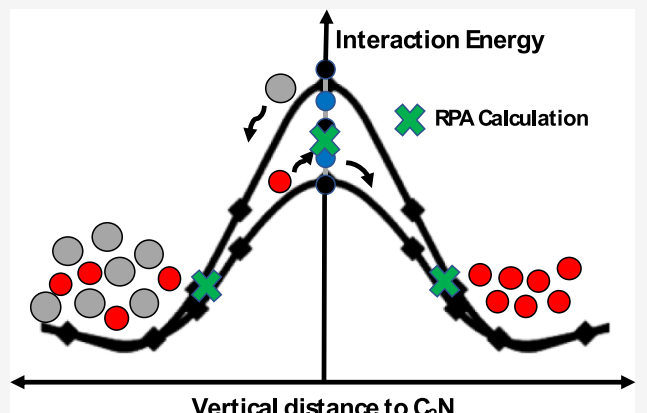
The comparison indicates that the interaction energy is less accurate with vdW density functional theory. On the other hand, more empirical corrections work reasonably well, a finding that we also confirm for another carbon nitride lattice, poly(triazine imide). Overall, we recommend these for screening carbon nitride materials for gas separation, but also comparing functionals with higherorder approaches when dealing with different materials.

\section{INTRODUCTION}

Energy-efficient separation of $\mathrm{He}$ and $\mathrm{H}_{2}$ is highly desirable for a number of medical, scientific, and energy applications. ${ }^{1,2}$ Currently, $\mathrm{He}$ is recovered from various gas streams $\left(\mathrm{CH}_{4}, \mathrm{~N}_{2}\right.$, etc.) using cryogenic distillation, ${ }^{3}$ while $\mathrm{H}_{2}$ is primarily produced by steam reforming and is purified from cogenerated $\mathrm{CO}_{2}$ by using $\mathrm{CO}_{2}$-selective solvents or adsorbents. ${ }^{4}$ These separation methods rely primarily on thermal energy, which makes them energy-intensive. As an alternative, high-performance membranes can reduce the energy footprint of these separation processes in remarkable ways. ${ }^{5}$ Therefore, screening and identifying materials that can provide high-performance $\mathrm{He}$ and $\mathrm{H}_{2}$ separation has become exceedingly important. ${ }^{6-12}$

Two-dimensional materials, especially those with a porous framework, are ideal for realizing membranes with an extremely thin selective layer. Among these, atom-thick materials hosting holes of the order of $3 \AA$ (when measured with respect to the electron density) can be very attractive for high-permeance $\mathrm{He}$ and $\mathrm{H}_{2}$ separation. In this case, gas transport across the selective layer is essentially given by just one crossing through the nanopore. The transport rate is then determined by the energy barrier at the nanopore ${ }^{13,14}$ and the surface concentration of molecules, which, in turn, is determined by the adsorption energy at the pore. A prominent candidate for two-dimensional membranes is nanoporous single-layer graphene, where the lattice is etched to incorporate A-scale nanopores for selective gas permeation. ${ }^{15-17}$ Another promising class of atom-thick materials for $\mathrm{He}$ and $\mathrm{H}_{2}$ separation is graphitic carbon nitrides. Several structures from this framework have intrinsic porosity with a pore size suitable for gas sieving. Among them, $\mathrm{C}_{2} \mathrm{~N}^{18}$ and triazine- and heptazine-based carbon nitride [i.e., poly(triazine imide) or $\mathrm{PTI}^{19,20}$ and poly(heptazine) imide or $\mathrm{PHI}^{21}$ are very interesting. All of them are atom-thick, and possess a high pore density and ordered porosity. However, the pore size and shape are different, $\mathrm{C}_{2} \mathrm{~N}$ has a rounded pore, whereas PTI and $\mathrm{PHI}$ have triangular pores.

$\mathrm{C}_{2} \mathrm{~N}$ belongs to the family of graphitic carbon nitrides, ${ }^{18}$ and hosts precisely shaped nanopores formed by the imide-linkage of six-membered $\mathrm{sp}^{2}$-carbon rings (Figure 1a). As a result, its lattice is highly porous (pore density $=1.7 \times 10^{14} \mathrm{~cm}^{-2}$ ), which makes it promising for realizing large gas permeance.

Received: April 28, 2021

Revised: July 12, 2021

Published: August 18, 2021 
(a)

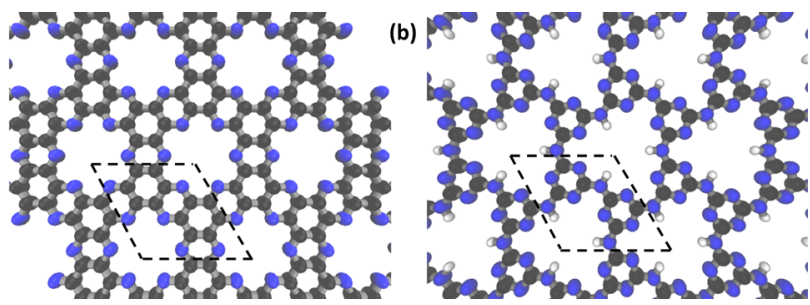

Figure 1. Ordered two-dimensional nanoporous structures of (a) $\mathrm{C}_{2} \mathrm{~N}$ and (b) PTI lattices. One can fit a circle with a diameter of $3.1 \AA$ in $\mathrm{C}_{2} \mathrm{~N}$ nanopores. The PTI nanopore has the shape of an equilateral triangule in which a circle with a diameter of $3.4 \AA$ can be fitted. The rhombi overlayed on their structure highlight the unit-cell for each structure.

The small electron density gap $(\sim 3.1 \AA)$ in $\mathrm{C}_{2} \mathrm{~N}$ nanopores is highly suited for selective $\mathrm{He}$ and $\mathrm{H}_{2}$ transport. In fact, several molecular simulation studies have indicated that only small gas molecules $\left(\mathrm{He}^{22}\right.$ and $\left.\mathrm{H}_{2}^{23}\right)$ can cross the $\mathrm{C}_{2} \mathrm{~N}$ nanopore with a low energy barrier. As a result, high $\mathrm{He} / \mathrm{CH}_{4}$ and $\mathrm{H}_{2} / \mathrm{N}_{2}$ selectivities can be achieved from the $\mathrm{C}_{2} \mathrm{~N}$ nanopores. Using van der Waals (vdW) density functional theory (DFT) calculations, Zhu et al. ${ }^{22}$ estimated a low energy barrier $(0.13 \mathrm{eV})$ for $\mathrm{He}$ and much larger energy barriers for $\mathrm{N}_{2}(0.87$ $\mathrm{eV})$ and $\mathrm{CH}_{4}(2.03 \mathrm{eV})$. This difference is large enough to deliver an exceptionally high gas-pair selectivity. Using classical molecular dynamics simulations, the same authors predicted a large He permeance $\left(10^{7}\right.$ gas permeance units or GPU; $1 \mathrm{GPU}$ $\left.=3.35 \times 10^{-10} \mathrm{~mol} \mathrm{~m}^{-2} \mathrm{~s}^{-1} \mathrm{~Pa}^{-1}\right)$ at $300 \mathrm{~K}$. This permeance is several orders of magnitude higher than those of commercial membranes for $\mathrm{He}$ separation. In a separate DFT study focusing on $\mathrm{H}_{2}$ transport across $\mathrm{C}_{2} \mathrm{~N}$, Xu et al. ${ }^{23}$ estimated energy barriers of $0.18,0.72$, and $1.05 \mathrm{eV}$ for $\mathrm{H}_{2}, \mathrm{CO}_{2}$, and $\mathrm{CO}$, respectively, indicating that $\mathrm{C}_{2} \mathrm{~N}$ blocks $\mathrm{CO}_{2}$ and $\mathrm{CO}$ transport, and selectively permeates $\mathrm{H}_{2}$. These molecular dynamic simulations also predicted an attractive $\mathrm{H}_{2}$ permeance of $10^{6}$ GPU. Such studies underscore the high impact that $\mathrm{C}_{2} \mathrm{~N}$ nanopores can have for an efficient separation of $\mathrm{He}$ and $\mathrm{H}_{2}$.

We note that the vdW-DFT literature on gas transport across $\mathrm{C}_{2} \mathrm{~N}$ nanopores is not consistent. Widely different energy barriers for $\mathrm{CO}_{2}$ have been reported, from $0.66^{22}$ to $0.72^{23}$ and $1.121 \mathrm{eV}^{24}$ Additionally, widely different minimum energy pathways have also been reported. For example, $\mathrm{Xu}$ et $\mathrm{al}^{23}$ and $\mathrm{Zhu}$ et $\mathrm{al}^{22}$ showed that the repulsive interaction between $\mathrm{CO}_{2}$ and the $\mathrm{C}_{2} \mathrm{~N}$ monolayers is maximized when the $\mathrm{C}$ atom of $\mathrm{CO}_{2}$ arrives at the center of the pore. In contrast, Deng et al. $^{24}$ concluded that the maximum repulsive interaction occurs when an $\mathrm{O}$ atom of $\mathrm{CO}_{2}$ is at the center of the pore. These differences are significant and raise questions on the accuracy of the vdW-DFT methods used to calculate the gas-lattice interactions. The role of vdW approximations in these studies is of particular interest. For example, Xu et al. ${ }^{23}$ and Deng et al. ${ }^{24}$ used vdW-DFT, ${ }^{25}$ while Zhu et al. $^{22}$ used a semiempirical Grimme-D2 correction. ${ }^{26}$ Interestingly, the Grimme approach ${ }^{27}$ has also been used to study the adsorption and desorption of $\mathrm{CO}_{2}$ on the surface of a $\mathrm{C}_{2} \mathrm{~N}$ monolayer. ${ }^{28}$ In addition to the aforementioned studies, $\mathrm{vdW}-\mathrm{DF} 2^{29}$ was used in the studies involving the electronic structure calculation of $\mathrm{C}_{2} \mathrm{~N}$ by Guan et al. ${ }^{30}$ and Bai et al. ${ }^{31}$

Here, we systematically investigate the influence of five vdW approximations [vdW-DF2, Grimme-D2, Grimme-D3, revised version of Vydrov and van Voorhis functional (rVV10), and Tkatchenko-Scheffler (TS)] on the interaction of gases with the $\mathrm{C}_{2} \mathrm{~N}$ lattice to predict the potential energy surface (PES) of $\mathrm{He}, \mathrm{H}_{2}, \mathrm{CO}_{2}$, and $\mathrm{N}_{2}$ on a $\mathrm{C}_{2} \mathrm{~N}$ nanopore. We compare these calculations using a more accurate technique, that is, the exact exchange (EXX) in conjugation with random-phase approximation (RPA) to show that the Grimme-D2, Grimme-D3, and TS approaches work reasonably well, whereas vdW-DF2 and rvv10 either overestimate or underestimate the interaction energy. This observation is also confirmed for another promising graphitic carbon nitride material, poly(triazine imide) or PTI. Overall, we argue that Grimme-D2, GrimmeD3, and TS are good choices to screen carbon nitride materials for gas separation.

\section{METHODS AND MODELS}

The vdW-DFT calculations are performed using the Quantum ESPRESSO. ${ }^{32,33}$ For the plane wave expansion of the wavefunctions, an energy cutoff of $60 \mathrm{Ry}$ is used. A $2 \times 2$ supercell is used to decouple in-plane molecule-molecule interactions. The Brillouin zone is sampled with a uniform $6 \times$ $6 \times 1$ unshifted $k$-point grids. To prevent any interactions among adjacent sheets, a vacuum region of $40 \AA$ is used in the $z$-direction. Calculations are performed with ultrasoft pseudopotentials $^{34,35}$ and a kinetic energy cutoff of $480 \mathrm{Ry}$ on the charge density. Five different dispersion corrections are used to include the contributions of dispersion interactions: vdWDF2, ${ }^{29}$ Grimme-D2, ${ }^{26}$ Grimme-D3, ${ }^{36}$ rVV10, $^{37,38}$ and TS. ${ }^{39}$ Because TS has not been implemented with ultrasoft pseudopotentials in Quantum ESPRESSO, TS calculations are carried out with norm-conserving pseudopotentials, ${ }^{40}$ using an energy cutoff of $100 \mathrm{Ry}$ for the wavefunctions.

We compare these vdW-DFT calculations using the adiabatic connection fluctuation-dissipation theorem (ACFDT) framework. ${ }^{41,42} \mathrm{We}$ obtain total energies with $\mathrm{EXX} / \mathrm{RPA}$, which is known to be accurate in capturing vdW interactions, and used it to find the binding energies for different types of molecules. ${ }^{4-46}$ The EXX/RPA calculations are performed using the implementation of the ACFDT formalism in the Quantum ESPRESSO distribution. ${ }^{32,46,47}$ Because the computational resources required for EXX/RPAbased calculations are significantly higher than those needed using conventional DFT, additional convergence tests are carried out (Figure S2) to use EXX/RPA in a more efficient manner. The PES of molecules is explored as a function of the relaxation of molecules (Figure S2a), supercell size (Figure $\mathrm{S} 2 \mathrm{~b}$ ), the density of the $k$-point grids (Figure S2c), the types of pseudopotentials (norm-conserving pseudopotential and ultrasoft pseudopotential, Figure S2d), and the cutoff values (Figure S2e). We show that EXX/RPA calculations on the gas-lattice systems considered in this study can be performed on a unit-cell using norm-conserving pseudopotential and sampling only at the $\Gamma$-point.

In this work, we use the EXX/RPA as an accurate and stateof-the-art technique to compare with our vdW-functional calculations. However, it is worth mentioning that the EXX/ RPA has its own limitations that have been addressed in several ways and at different levels of sophistication. These include semilocal and non-local corrections ${ }^{48,49}$ in order to describe short-range correlations properly, the use of improved kernel from TDDFT such as the renormalized adiabatic-LDA $\operatorname{kerne}^{50,51}$ or the exact-exchange kernel ${ }^{52-54}$ to describe better the density-density response function, or corrections based on many-body perturbation theory such as the screened-second order exchange $e^{55-57}$ or the single-excitation correction. ${ }^{58,59}$ 
We also mention that all these corrective schemes have also been applied in a range-separated framework, ${ }^{60,61}$ where only the long-range behavior of the RPA (and beyond schemes) is retained. In contrast, the short-range behavior is replaced by the one from semilocal or hybrid functionals. It has recently been shown that important effects beyond the dipole approximation typical of the RPA can be included within the many-body dispersion framework ${ }^{62}$ by accounting for the dipole-correlated Coulomb singles, ${ }^{63}$ which turns out to be particularly relevant in supramolecular systems and under nanoconfinement.

\section{RESULTS AND DISCUSSION}

Both $\mathrm{C}_{2} \mathrm{~N}$ and PTI have two-dimensional hexagonal lattices. To find the minimum energy configuration (lattice parameters), we relaxed the framework of $\mathrm{C}_{2} \mathrm{~N}$ and PTI using the five vdW approximations (vdW-DF2, Grimme-D2, Grimme-D3, TS, and rVV10) considered in this work. The relaxed lattice parameters (Table 1) go from 8.309 to $8.331 \AA$ for $\mathrm{C}_{2} \mathrm{~N}$ and

Table 1. Relaxed $\mathrm{C}_{2} \mathrm{~N}$ and PTI Lattice Parameters Using the Five vdW Approximations

$\begin{array}{lcc}\begin{array}{c}\text { vdW dispersion } \\ \text { correction }\end{array} & \begin{array}{c}\mathrm{C}_{2} \mathrm{~N} \text { lattice parameter } \\ (\AA)\end{array} & \begin{array}{c}\text { PTI lattice parameter } \\ (\AA)\end{array} \\ \text { vdW-DF2 } & 8.309 & 8.638 \\ \text { Grimme-D2 } & 8.318 & 8.642 \\ \text { Grimme-D3 } & 8.316 & 8.645 \\ \text { TS } & 8.317 & 8.647 \\ \text { rVV10 } & 8.331 & 8.673\end{array}$

8.638 to $8.673 \AA$ for PTI, in agreement with the literature. ${ }^{22,23,64}$ Next, we analyze the effective pore size for gas transport in these two lattices. The $\mathrm{C}_{2} \mathrm{~N}$ nanopores are somewhat round in shape. The effective pore size is determined by the six $\mathrm{N}$ atoms (blue atoms in Figure 1a) protruding in the nanopore. One can fit a circle with a diameter of $3.1 \AA$ in this nanopore. On the other hand, the PTI nanopore has a triangular shape (Figure $1 \mathrm{~b}$ ), where one can fit a circle with a diameter of $3.4 \AA$. Next, we systematically investigate the role of the five vdW approximations in the gaslattice interactions.

Constrained relaxation calculations are performed to study the interactions of $\mathrm{He}, \mathrm{H}_{2}, \mathrm{CO}_{2}$, and $\mathrm{N}_{2}$ with the nanoporous lattice as a function of the distance between the molecule and the lattice. For this, the lattice is placed in the $x-y$ plane at $z=$ 0 , and the gas molecule is placed at a certain distance along the $z$-direction. The center of mass of the molecule is fixed in the $z$-direction, but the molecule is allowed to relax in the $x-y$ plane. In this way, the molecule has the freedom to move in $x$ and $y$ directions to minimize the potential at a given distance from the lattice. Convergence thresholds of $2 \times 10^{-6} \mathrm{Ry}$ and $10^{-4} \mathrm{Ry} / \mathrm{Bohr}$ for the total energy and forces, respectively, are used. An assumption of a flexible or a rigid lattice did not significantly change the PES (Figure S1); therefore, we carried out the simulations using the rigid lattice.

To investigate the minimum energy pathway for the gas transport through the nanopore, the interaction between the gas molecule and the lattice is calculated as follows

$$
E_{\text {int }}=E_{\text {gas }+ \text { lattice }}-E_{\text {gas }}-E_{\text {lattice }}
$$
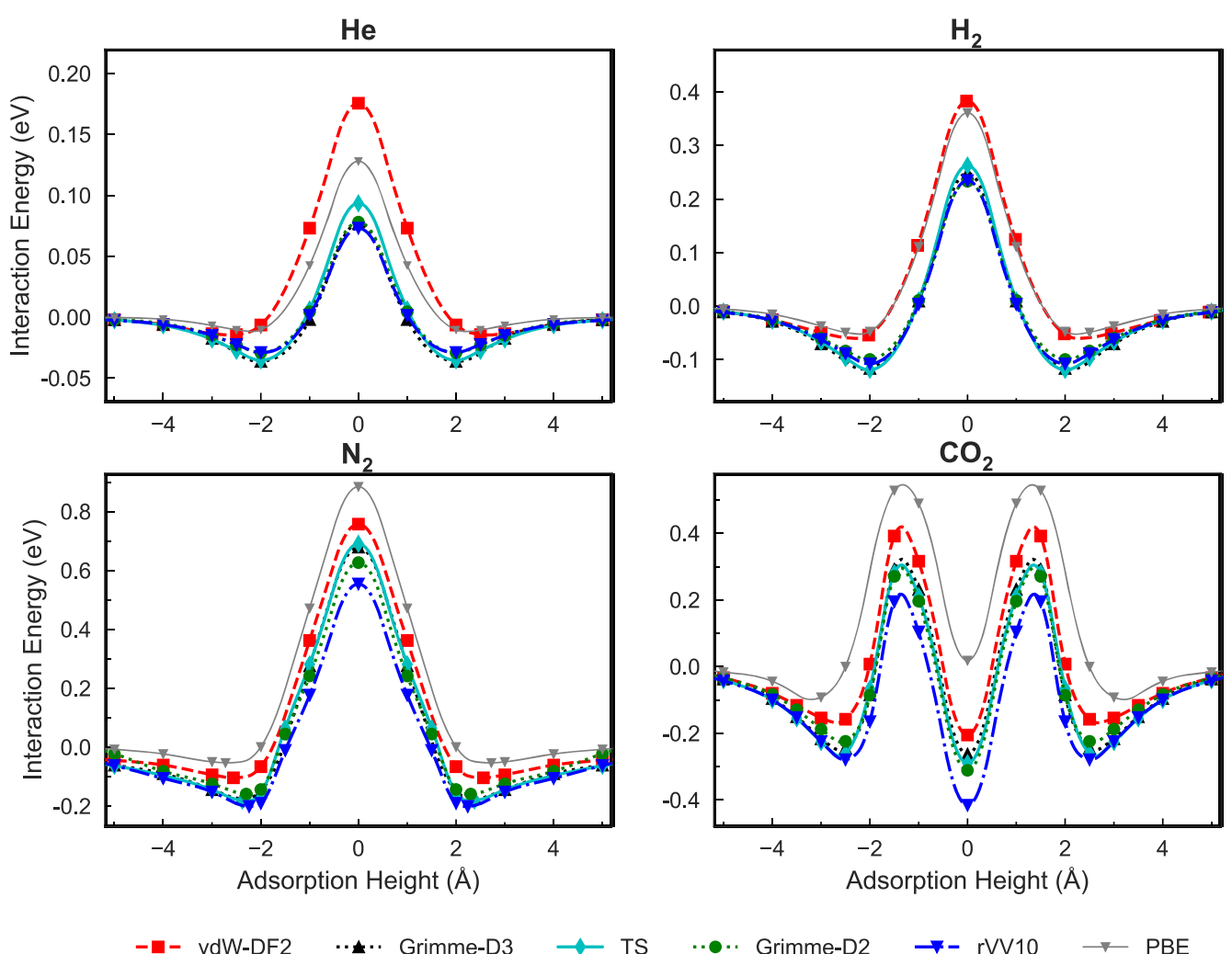

TS

.•.. Grimme-D2 $\rightarrow-$ rVV10 $\rightarrow$ PBE

Figure 2. PESs of $\mathrm{He}$ (top left), $\mathrm{H}_{2}$ (top right), $\mathrm{N}_{2}$ (bottom left), and $\mathrm{CO}_{2}$ (bottom right) on the $\mathrm{C}_{2} \mathrm{~N}$ lattice calculated using the five vdW approximations (vdW-DF2, Grimme-D2, Grimme-D3, rVV10, and TS) and the PBE functional. 
where $E_{\text {gastlattice }}$ is the total energy of the interacting system. $E_{\text {gas }}$ and $E_{\text {lattice }}$ are the total energy of the gas and the lattice, respectively, when they are isolated and do not interact with each other. Because the PES for the gases considered in this study is simple enough ( $\mathrm{He}$ is a single atom; $\mathrm{H}_{2}, \mathrm{~N}_{2}$, and $\mathrm{CO}_{2}$ are linear molecules), it is not required to carry out nudge elastic band calculations, and the constrained relaxation is sufficient.

The PES of the four gases on the $\mathrm{C}_{2} \mathrm{~N}$ lattice calculated using vdW-DF2, Grimme-D2, Grimme-D3, rVV10, and TS are shown in Figure 2, and are compared to the PES calculated using the Perdew-Burke-Ernzerhof (PBE) functional. Following observations are common to all PES:

(i) All four molecules prefer to adsorb on the $\mathrm{C}_{2} \mathrm{~N}$ lattice. The center of the mass of the adsorbed species lies in the centrosymmetric $z$-axis as shown in the Supporting Information Figure S3, in all four cases. The height of the adsorbed molecule above the pore, that is, the adsorption height, is listed in Table S1.

(ii) For the linear molecules $\left(\mathrm{H}_{2}, \mathrm{CO}_{2}\right.$, and $\left.\mathrm{N}_{2}\right)$, the most favorable configuration in the absorbed state is horizontal (configuration "1" in Figure 3).

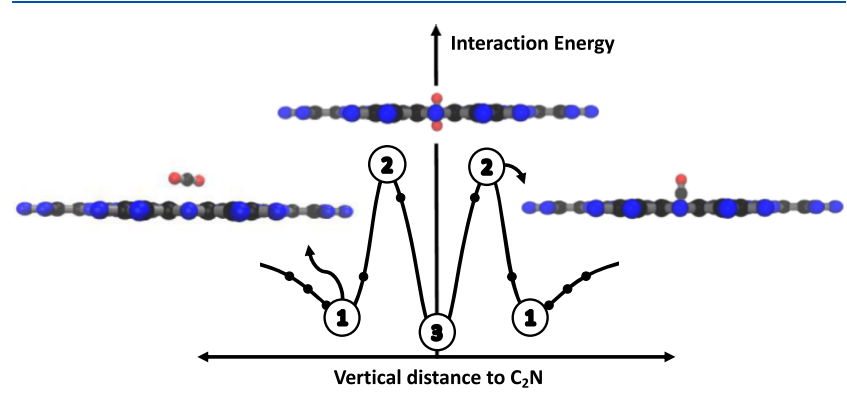

Figure 3. $\mathrm{CO}_{2}$ configurations at three different $z$ positions above the $\mathrm{C}_{2} \mathrm{~N}$ nanopore. In the first configuration, the $\mathrm{CO}_{2}$ adsorbs horizontally above the nanopore. As it moves closer to the nanopore, it orients vertically (configuration " 2 "). Here, the $\mathrm{O}$ atom of the $\mathrm{CO}_{2}$ is at the center of the nanopore. In this case, the interaction energy is governed by the repulsive interactions between $\mathrm{O}$ and the $\mathrm{N}$ atoms of the $\mathrm{C}_{2} \mathrm{~N}$. Finally, $\mathrm{CO}_{2}$ arrives at the center of the nanopore maintaining its vertical orientation (configuration " 3 "), where the $\mathrm{C}$ atom of $\mathrm{CO}_{2}$ is at the center of the nanopore. (iii) To fit inside the nanopore, the linear molecules prefer to orient vertically (configurations " 2 " and " 3 " in Figure 3 ). A vertical position of linear molecules inside the pore matches common intuition because if the gases were in the horizontal position, there would be a relatively larger overlap between the gas and the electron cloud of the pore edge.

(iv) The total energy of the system increases when the gases are brought close to the nanopore from the adsorbed state. For $\mathrm{He}, \mathrm{H}_{2}$, and $\mathrm{N}_{2}$, the interaction energy increases monotonically as $z$ decreases. Consequently, their PES has only one maximum at $z=0$ (at the center of the nanopore). In contrast, the PES for $\mathrm{CO}_{2}$ shows a maximum away from the nanopore $(z \neq 0)$. This happens when the center of mass of $\mathrm{CO}_{2}(\mathrm{C}$ atom of $\mathrm{CO}_{2}$ ) is at a distance of $1.1 \AA$ from the nanopore (Figure 2). This is the position when the $\mathrm{O}$ atom of $\mathrm{CO}_{2}$ is at the center of the nanopore (the configuration " 2 " in Figure 3).

The resulting M-shaped PES of $\mathrm{CO}_{2}$ is unique. Deng et al. ${ }^{24}$ also report an M-shaped PES with vdW-DF2 approximation, but the interaction energies are approximately 2.5-fold higher in their case. Later, by comparison with EXX/RPA calculations, we show that the M-shaped curve and energetics calculated in this study are indeed quite accurate. We also note that $\mathrm{M}$-shaped PES for $\mathrm{CO}_{2}$ has been reported for gas transport across a $\mathrm{MoS}_{2}$ nanopore. ${ }^{65}$

Comparing several PES in Figure 2, it is evident that the PES calculated using the PBE functional underpredicts the attractive interactions between the molecule and the lattice compared to all other vdW functionals. Among the PES from the five vdW approximations, there is a significant difference in terms of the adsorption energy $\left(E_{\mathrm{ads}}\right.$, defined as the minimum $\left.E_{\text {int }}\right)$ and the energy barrier $\left(E_{\text {act }}\right.$ defined as the difference in $E_{\text {int }}$ at $z=0$ and $z$ corresponding to adsorption). $E_{\text {ads }}$ values for $\mathrm{He}, \mathrm{H}_{2}, \mathrm{~N}_{2}$, and $\mathrm{CO}_{2}$ are in the range of -0.01 to $-0.03,-0.06$ to $-0.11,-0.10$ to -0.20 , and -0.16 to $-0.28 \mathrm{eV}$, respectively. $E_{\text {act }}$ values for $\mathrm{He}, \mathrm{H}_{2}$, and $\mathrm{N}_{2}$ are in the range of $0.10-0.19$, $0.33-0.44$, and $0.76-0.87 \mathrm{eV}$, respectively.

The variation in $E_{\text {ads }}$ and $E_{\text {act }}$ from the five vdW approximations is expected to affect the $\mathrm{He}$ and $\mathrm{H}_{2}$ separation performance. To understand the influence of $\mathrm{vdW}$ approximations on the $\mathrm{He} / \mathrm{N}_{2}$ and $\mathrm{H}_{2} / \mathrm{N}_{2}$ selectivities, we estimate the approximate selectivities by calculating the ratio of gas flux
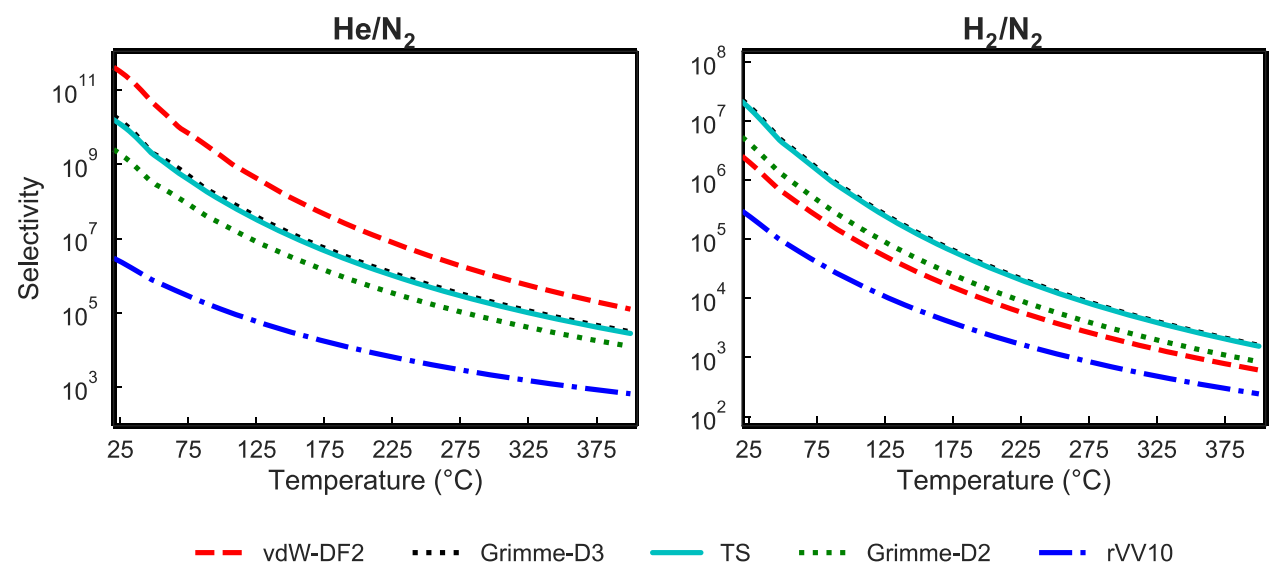

vdW-DF2 $\quad \ldots$ Grimme-D3

TS $\quad .$. Grimme-D2 -. rVV10

Figure 4. $\mathrm{He} / \mathrm{N}_{2}$ (left) and $\mathrm{H}_{2} / \mathrm{N}_{2}$ (right) selectivities from the $\mathrm{C}_{2} \mathrm{~N}$ nanopore as a function of temperature calculated with eq 3 using $E_{\text {act }}$ and $E_{\text {ads }}$ from the five vdW approximations. 

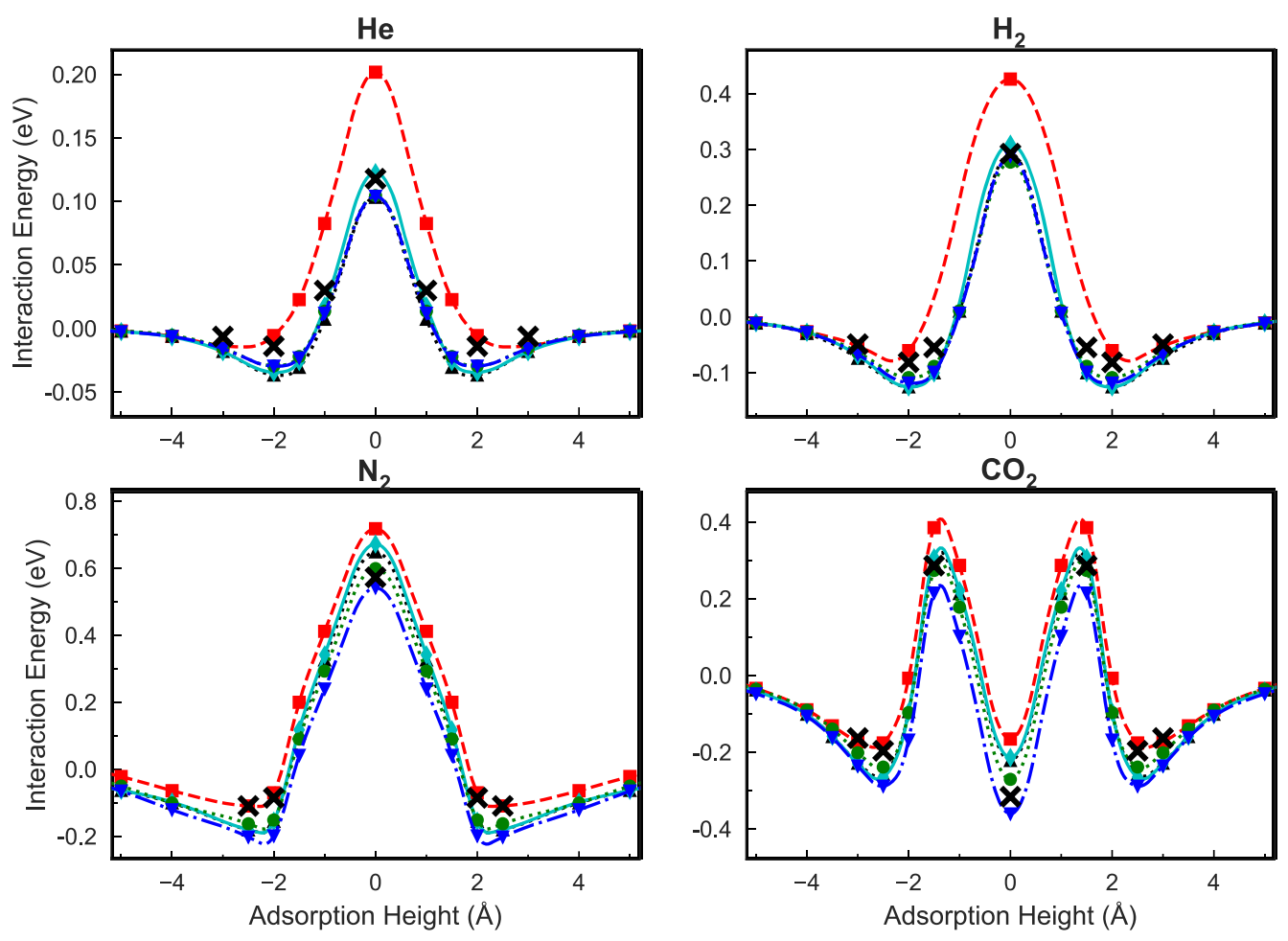

$-\square-v d W-D F 2 \quad \cdots \neq \cdot \cdot$ Grimme-D3 $\rightarrow$ TS

..* Grimme-D2 $\rightarrow-$ rVV10 $\quad$ R RPA

Figure 5. PESs of $\mathrm{He}, \mathrm{H}_{2}, \mathrm{~N}_{2}$, and $\mathrm{CO}_{2}$ on a $\mathrm{C}_{2} \mathrm{~N}$ monolayer with different vdW approximations, vdW-DF2, Grimme-D2, Grimme-D3, rVV10, and TS. vdW-DFT calculations are compared against the results calculated with the EXX/RPA method.

(eq 2). Briefly, the gas flux, $J$, through a nanopore of commensurate size can be obtained by the transition-state theory, which yields an Arrhenius-type relationship to the flux (eq 2), where the pre-exponential factor, $A$, represents an entropic loss of the gas upon adsorption. ${ }^{66}$ Given that (i) the energy barrier differences between $\mathrm{He}\left(\right.$ or $\mathrm{H}_{2}$ ) and $\mathrm{N}_{2}$ are quite significant in the present case, and (ii) the entropic terms for light gases are generally within an order of magnitude, ${ }^{67,68}$ we can neglect the entropic contribution to estimate the selectivity (eq 3 ).

$$
\begin{aligned}
& \left.J_{\mathrm{i}} \propto \operatorname{Aexp}\left(-\left(E_{\mathrm{ads}, \mathrm{i}}+E_{\mathrm{act}, \mathrm{i}}\right)\right) / R T\right) \\
& S_{\mathrm{i} / \mathrm{j}} \sim \frac{\left.\exp \left(-\left(E_{\mathrm{ads}, \mathrm{i}}+E_{\mathrm{act}, \mathrm{i}}\right)\right) / R T\right)}{\left.\exp \left(-\left(E_{\mathrm{ads}, \mathrm{j}}+E_{\mathrm{act}, \mathrm{j}}\right)\right) / R T\right)} \\
& A=\frac{1}{L} \sqrt{\frac{R T}{2 \pi M}} \exp \left(\frac{\Delta S}{R}\right)
\end{aligned}
$$

where $E_{\mathrm{ads}, \mathrm{i}}$ and $E_{\mathrm{ads}, \mathrm{j}}$ represent $E_{\mathrm{ads}}$ for molecules $\mathrm{i}$ and $\mathrm{j}$, respectively. Similarly, $E_{\text {act, }, \mathrm{i}}$ and $E_{\mathrm{act}, \mathrm{j}}$ represent $E_{\text {act }}$ for molecules $\mathrm{i}$ and j, respectively. $R$ is the universal gas constant and $T$ is the temperature. $L$ is the half-peak width of the probability distribution of the gas along the transport path from the adsorbed phase to the center of the pore, $\Delta S$ is the corresponding entropy change, and $M$ is the molecular weight of the gas. Based on this, an approximate gas-pair selectivity within the temperature range of $25-400{ }^{\circ} \mathrm{C}$ is presented in Figure 4. In general, all five vdW approximations predict that $\mathrm{C}_{2} \mathrm{~N}$ nanopore would yield extremely high $\mathrm{H}_{2} / \mathrm{N}_{2}$ and $\mathrm{He} / \mathrm{N}_{2}$ selectivities, which make $\mathrm{C}_{2} \mathrm{~N}$ highly promising for these separations. The selectivity reduces with temperature in all cases because the sum of $E_{\text {ads }}$ and $E_{\text {act }}$ also known as apparent activation energy in the experimental studies, is higher for $\mathrm{N}_{2}$ than those for $\mathrm{He}$ and $\mathrm{H}_{2}$, because of much higher $E_{\text {act }}$ for $\mathrm{N}_{2}$.

The discrepancy between the predicted selectivities from the five vdW approximations is quite large, differing by several orders of magnitude (Figure 4). Having a reliable quantitative estimation of the selectivity is especially important for $\mathrm{H}_{2} / \mathrm{N}_{2}$ separation at high temperature, relevant to $\mathrm{H}_{2}$ production for $\mathrm{NH}_{3}$ synthesis, ${ }^{69}$ where selectivities of 100 and 1000 can lead to a large difference in membrane process design. To understand which of these vdW approximations provide the most accurate description of PES, we compared our calculations using a more advanced technique, EXX/RPA, which is known to accurately capture dispersion interactions ${ }^{43}$ (Figure 5). For example, EXX/RPA calculations have shown a good agreement with experimental data on physisorption energies for several molecules on various surfaces. ${ }^{44-46,57,70-72}$

By comparing PES from the five vdW approximations with the interaction energy data from EXX/RPA calculations, we can make the following observations:

(i) vdW-DF2 correctly predicts the adsorption energy but overpredicts the repulsive interaction energy at the center of the pore.

(ii) rVV10 overpredicts the gas-lattice attractive interactions. In some cases, it also underpredicts the repulsive interaction energy at the center of the pore.

(iii) Grimme-D2, Grimme-D3, and TS slightly underpredict the adsorption energy but correctly predict the repulsive interaction at the center of the pore.

The EXX/RPA calculations validate the M-shaped PES of $\mathrm{CO}_{2}$ on the $\mathrm{C}_{2} \mathrm{~N}$ nanopore and confirm the argument by Deng et $\mathrm{al}^{24} \mathrm{We}$ also hold the view that the maximum energy at configuration " 2 " is due to the strong repulsive interactions 

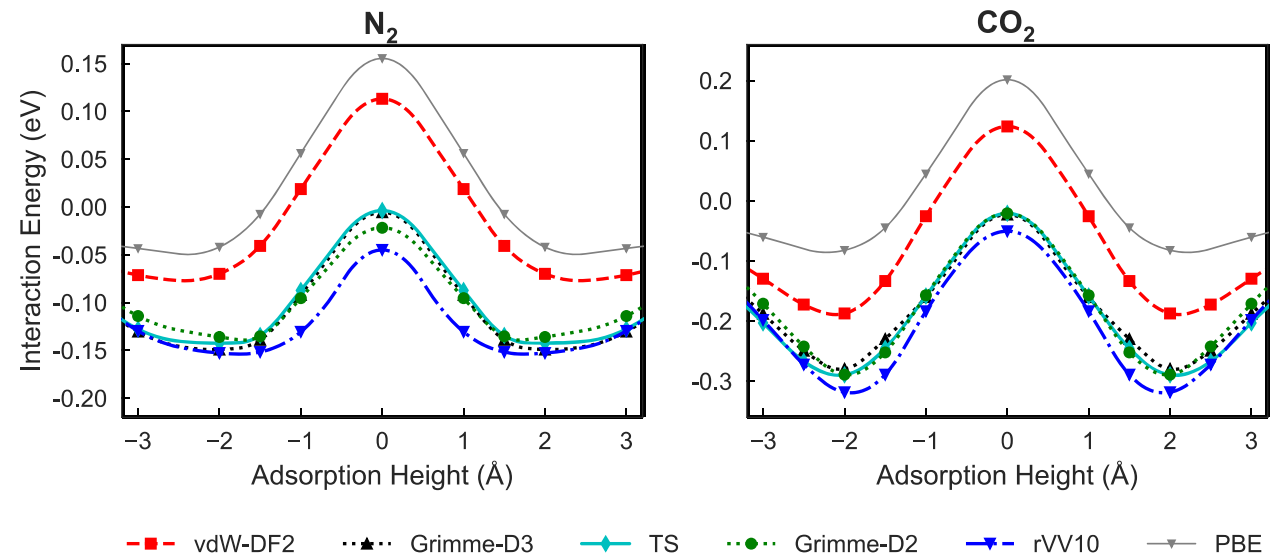

$-1-\mathrm{vdW}-\mathrm{DF} 2 \quad \cdots \neq \cdot$ Grimme-D3 $\rightarrow$ TS

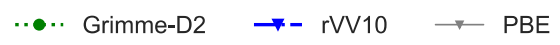

Figure 6. PESs of $\mathrm{N}_{2}$ (left) and $\mathrm{CO}_{2}$ (right) on a PTI nanopore using the five vdW approximations and the PBE functional.
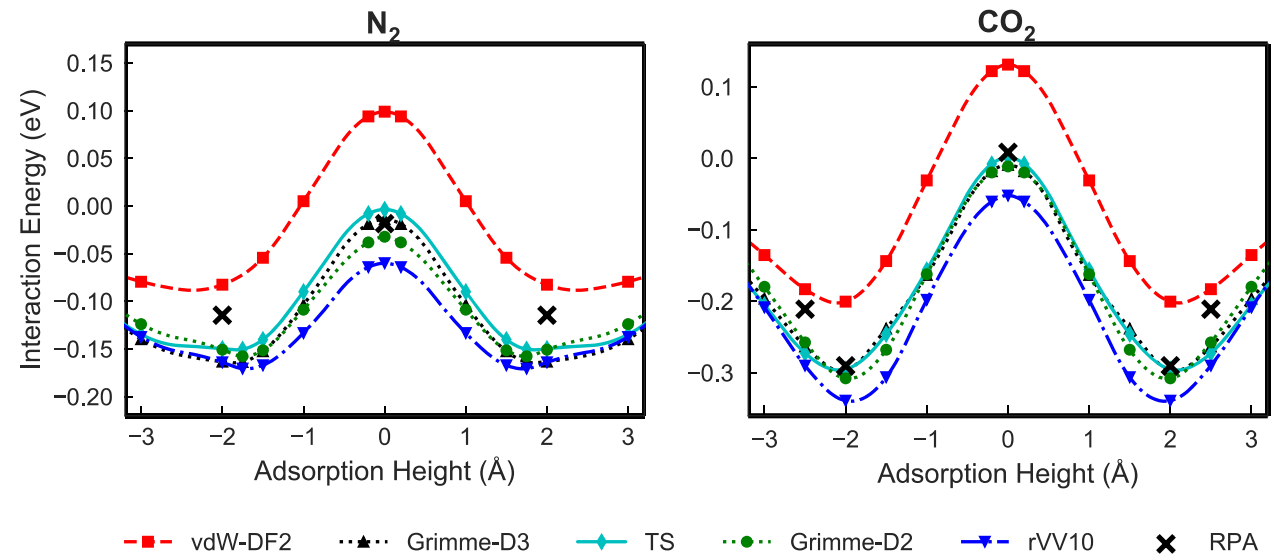

Figure 7. PESs of $\mathrm{N}_{2}$ and $\mathrm{CO}_{2}$ on a PTI monolayer with five vdW approximations compared against interaction energy data from EXX/RPA calculations.

between the $\mathrm{O}$ atom of $\mathrm{CO}_{2}$ and the $\mathrm{N}$ atoms of the nanopore when $\mathrm{CO}_{2}$ enters the nanopore in the vertical orientation with $\mathrm{O}$ atom arriving at the center of the pore. When the $\mathrm{C}$ atom of $\mathrm{CO}_{2}$ finally arrives at the center of the pore (configuration " 3 "), the overall repulsive interaction reduces as a result of additional attraction interactions between the two $\mathrm{O}$ atoms of $\mathrm{CO}_{2}$, positioned at the two sides of the nanopore, and the $\mathrm{N}$ atoms of the pore.

The above findings on the effect of vdW approximations on the PES of gas molecules motivated us to extend our analysis to PTI nanopores, another promising two-dimensional nanoporous material for high-flux gas separation. PTI nanopores are triangular in shape and are built from imide linkages between six triazine units (Figure 1). Our recent simulation study has shown that PTI nanopores can be used for sieving $\mathrm{Kr}$ (a kinetic diameter of $3.60 \AA$ ) from Xe (a kinetic diameter of $3.96 \AA$ ) with a large $\mathrm{Kr} / \mathrm{Xe}$ separation selectivity and an attractive $\mathrm{Kr}$ permeance. ${ }^{68}$ Here, we explore the PES of $\mathrm{N}_{2}$ and $\mathrm{CO}_{2}$ on PTI with the same set of $\mathrm{vdW}$ approximations as that used for $\mathrm{C}_{2} \mathrm{~N}$.

Figure 6 compares six PES (one for each density functional) for $\mathrm{N}_{2}$ and $\mathrm{CO}_{2}$ on a PTI nanopore. Again, in all cases, in terms of the orientation of the gas molecules as a function of $z$, we observe a similar behavior to the one observed in the case of $\mathrm{C}_{2} \mathrm{~N}$, that is, both $\mathrm{N}_{2}$ and $\mathrm{CO}_{2}$ prefer to adsorb in the horizontal configuration, whereas they prefer to enter the nanopore in the vertical configuration. Again, PBE underestimates the attractive interactions compared to all the other vdW functionals. We also observe quantitatively different interaction energies $\left(E_{\text {ads }}\right.$ and $\left.E_{\text {act }}\right)$ for the five vdW approximations. $E_{\text {ads }}$ values for $\mathrm{N}_{2}$ and $\mathrm{CO}_{2}$ are in the range of -0.07 to -0.15 and -0.19 to $-0.32 \mathrm{eV}$, respectively. $E_{\text {act }}$ values for $\mathrm{N}_{2}$ and $\mathrm{CO}_{2}$ are in the range of $0.11-0.19$ and $0.26-$ $0.31 \mathrm{eV}$, respectively. We find it interesting that $E_{\text {act }}$ for $\mathrm{CO}_{2}$ is higher than that for $\mathrm{N}_{2}$ given that $\mathrm{CO}_{2}$ has a smaller kinetic diameter than $\mathrm{N}_{2}(3.30$ vs $3.64 \AA)$. In the case of $\mathrm{C}_{2} \mathrm{~N}$, the $E_{\text {act }}$ value for $\mathrm{CO}_{2}$ was smaller than that of $\mathrm{N}_{2}$. This unexpected result in the case of PTI is likely rooted in the much stronger attractive interaction (or the potential well) of $\mathrm{CO}_{2}$ at the adsorption site, which increases the energy barrier for $\mathrm{CO}_{2}$ to hop to the transition state at the center of the pore. Next, to understand the accuracy of our calculations, we compare the PES with the interaction energy data from the EXX/RPA method.

Figure 7 compares the PES of $\mathrm{N}_{2}$ and $\mathrm{CO}_{2}$ on PTI obtained with vdW-DFT calculations with the interaction energy data from EXX/RPA calculations. Again, we can make a few general observations:

(i) vdW-DF2 underestimates the gas-lattice attractive interactions and ends up overpredicting the repulsive interaction energy at the center of the pore by a large margin.

(ii) rVV10 overestimates the gas-lattice attractive interactions and ends up underpredicting the repulsive interaction energy at the center of the pore by a large margin. However, the deviation from the EXX/RPA results is lower than that for vdW-DF2. 
(iii) Grimme-D2, Grimme-D3, and TS slightly overestimate the attractive interactions for $\mathrm{N}_{2}$ but correctly predict it for $\mathrm{CO}_{2}$. They correctly predict the interaction at the center of the pore for both gases.

Overall, Grimme-D2, Grimme-D3, and TS lead to more accurate PES for PTI compared to those from vdW-DF2 and rVV10, consistent with the observations for $\mathrm{C}_{2} \mathrm{~N}$, and therefore, can be considered a good choice for calculating gas-lattice interaction from a wider material database of the carbon nitride framework.

\section{DISCUSSION ON THE LOWER ACCURACY OF VDW-DF2 AND RVV10}

Our investigation on two promising carbon nitride materials for gas separation, $\mathrm{C}_{2} \mathrm{~N}$ and PTI, reveals that vdW-DFT calculations using Grimme-D2, Grimme-D3, and TS functionals are fairly accurate in capturing dispersion interactions. On the other hand, vdW-DF2 tends to underestimate the gaslattice attractive interaction for both carbon nitride lattices. The same behavior is reported for carbon nanotubes when vdW-DF2 is used. ${ }^{73}$ On the other hand, rVV10 tends to overestimate the attractive interaction. The relatively poor performance of $\mathrm{rVV} 10$ can be attributed to the negligence of the charge screening effect, which is known to be very important for anisotropic systems such as those considered in our study. ${ }^{74,75}$ Moreover, it is known that the rVV10 performance improves when it is used with more "repulsive" semilocal functionals (those capturing the exchange repulsion). ${ }^{74}$ Therefore, the PBE functionals might not be the best option to be paired with rVV10 (in this study, we used the $\mathrm{PBE}$ exchange-correlation functional for the sake of consistency).

\section{CONCLUSIONS}

This study illustrates the importance of capturing accurate $\mathrm{vdW}$ interactions for screening nanoporous materials for gas separation. We compared PES of $\mathrm{He}, \mathrm{H}_{2}, \mathrm{~N}_{2}$, and $\mathrm{CO}_{2}$ across $\mathrm{C}_{2} \mathrm{~N}$ for five vdW approximations (Grimme-D2, Grimme-D3, rVV10, TS, and vdW-DF2), and showed that gas-pair selectivity changes significantly depending on the choice of the vdW approximation. By comparing vdW-DFT calculations against more accurate EXX/RPA calculations, we show that Grimme-D2, Grimme-D3, and TS are reliable choices to investigate carbon nitride nanopores for gas separation. We find a unique $\mathrm{M}$-shaped PES for $\mathrm{CO}_{2}$, which originates from initially repulsive to subsequently attractive interactions between the $\mathrm{O}$ atom of $\mathrm{CO}_{2}$ and the $\mathrm{N}$ atom of the nanopore when $\mathrm{CO}_{2}$ traverses the nanopore in the vertical orientation. The gas-pair selectivity estimates based on transition-statetheory confirm that $\mathrm{C}_{2} \mathrm{~N}$ is an excellent candidate for $\mathrm{He} / \mathrm{N}_{2}$ and $\mathrm{H}_{2} / \mathrm{N}_{2}$ separation, with selectivity estimated over 1000 over a wide range of temperatures.

The accuracy of vdW-DF2 and rvv10 might be affected by the anisotropic nature of the gas-lattice system. We note that the conclusions on the accuracy of Grimme-D2, Grimme-D3, and TS over vdW-DF2 and TS for carbon nitride frameworks remain to be verified for materials outside the carbon nitride family. The careful approach taken here for comparing the vdW approximations with EXX/RPA calculations can be applied to several other relevant materials, from nanoporous single-layer graphene to transition metal dichalcogenides and hexagonal boron nitride.

\section{ASSOCIATED CONTENT}

\section{(s) Supporting Information}

The Supporting Information is available free of charge at https://pubs.acs.org/doi/10.1021/acs.jpcc.1c03822.

Exploring the influence of fixing the monolayer during the PES calculations; RPA convergence tests; $\mathrm{N}_{2}$ molecule on top of the $\mathrm{C}_{2} \mathrm{~N}$ monolayer; adsorption height of $\mathrm{H}_{2}, \mathrm{He}, \mathrm{N}_{2}$, and $\mathrm{CO}_{2}$ on a $\mathrm{C}_{2} \mathrm{~N}$ monolayer; and vibrational frequency of adsorbed $\mathrm{H}_{2}$ on the $\mathrm{C}_{2} \mathrm{~N}$ monolayer (PDF)

\section{AUTHOR INFORMATION}

\section{Corresponding Authors}

Nicola Marzari - Theory and Simulation of Materials (THEOS) and National Centre for Computational Design and Discovery of Novel Materials (MARVEL), EPFL, CH1015 Lausanne, Switzerland; (1) orcid.org/0000-00029764-0199; Email: nicola.marzari@epfl.ch

Kumar Varoon Agrawal - Laboratory of Advanced Separations (LAS), Ecole Polytechnique Fédérale de Lausanne (EPFL), CH-1950 Sion, Switzerland; () orcid.org/0000-0002-5170-6412; Email: kumar.agrawal@epfl.ch

\section{Authors}

Mohammad Tohidi Vahdat - Laboratory of Advanced Separations (LAS), École Polytechnique Fédérale de Lausanne (EPFL), CH-1950 Sion, Switzerland; Theory and Simulation of Materials (THEOS) and National Centre for Computational Design and Discovery of Novel Materials (MARVEL), EPFL, CH-1015 Lausanne, Switzerland

Davide Campi - Theory and Simulation of Materials (THEOS) and National Centre for Computational Design and Discovery of Novel Materials (MARVEL), EPFL, CH1015 Lausanne, Switzerland; Present

Address: Dipartimento di Scienza dei Materiali, Università di Milano-Bicocca, Via R. Cozzi 55, I-20125 Milano, Italy; (i) orcid.org/0000-0002-6278-4352

Nicola Colonna - Theory and Simulation of Materials (THEOS) and National Centre for Computational Design and Discovery of Novel Materials (MARVEL), EPFL, CH1015 Lausanne, Switzerland; Laboratory for Neutron Scattering and Imaging (LNS), Paul Scherrer Institute (PSI), CH-5232 Villigen, Switzerland; ㅇo이.org/0000-00026106-6316

Complete contact information is available at:

https://pubs.acs.org/10.1021/acs.jpcc.1c03822

\section{Notes}

The authors declare no competing financial interest.

\section{ACKNOWLEDGMENTS}

We thank our host institution, EPFL, for generous support. Parts of the work were funded by the Swiss National Science Foundation (Assistant Professor Energy Grant; grant no PYAPP2_173645). We thank the Swiss National Supercomputing Center, CSCS, for the allocation of computing resources (production projects s860 and s953). D.C. and N.M. acknowledge support from the MARVEL NCCR. D.C. acknowledges support from the EPFL Fellows program cofunded by Marie Sklodowska-Curie, Horizon 2020 grant agreement no. 66566 . 


\section{REFERENCES}

(1) Schrier, J. Helium Separation Using Porous Graphene Membranes. J. Phys. Chem. Lett. 2010, 1, 2284-2287.

(2) Marbán, G.; Valdés-Solís, T. Towards the hydrogen economy? Int. J. Hydrogen Energy 2007, 32, 1625-1637.

(3) Van Sciver, S. W. Helium Cryogenics, 2nd ed.; International Cryogenics Monograph Series; Springer: New York, 2012.

(4) Gallucci, F.; Fernandez, E.; Corengia, P.; van Sint Annaland, M. Recent Advances on Membranes and Membrane Reactors for Hydrogen Production. Chem. Eng. Sci. 2013, 92, 40-66.

(5) National Academies of Sciences, Engineering, and Medicine, Division on Earth and Life Studies, Board on Chemical Sciences and Technology, Committee on a Research Agenda for a New Era in Separation Science A Research Agenda for Transforming Separation Science; National Academies Press, 2019.

(6) Shan, M.; Xue, Q.; Jing, N.; Ling, C.; Zhang, T.; Yan, Z.; Zheng, $\mathrm{J}$. Influence of Chemical Functionalization on the $\mathrm{CO}_{2} / \mathrm{N}_{2}$ Separation Performance of Porous Graphene Membranes. Nanoscale 2012, 4, 5477.

(7) Tao, Y.; Xue, Q.; Liu, Z.; Shan, M.; Ling, C.; Wu, T.; Li, X. Tunable Hydrogen Separation in Porous Graphene Membrane: FirstPrinciple and Molecular Dynamic Simulation. ACS Appl. Mater. Interfaces 2014, 6, 8048-8058.

(8) Du, H.; Li, J.; Zhang, J.; Su, G.; Li, X.; Zhao, Y. Separation of Hydrogen and Nitrogen Gases with Porous Graphene Membrane. J. Phys. Chem. C 2011, 115, 23261-23266.

(9) Jiang, D.-e.; Cooper, V. R.; Dai, S. Porous Graphene as the Ultimate Membrane for Gas Separation. Nano Lett. 2009, 9, 40194024.

(10) Wu, T.; Xue, Q.; Ling, C.; Shan, M.; Liu, Z.; Tao, Y.; Li, X. Fluorine-Modified Porous Graphene as Membrane for $\mathrm{CO}_{2} / \mathrm{N}_{2}$ Separation: Molecular Dynamic and First-Principles Simulations. J. Phys. Chem. C 2014, 118, 7369-7376.

(11) Ambrosetti, A.; Silvestrelli, P. L. Gas Separation in Nanoporous Graphene from First Principle Calculations. J. Phys. Chem. C 2014, $118,19172-19179$.

(12) Zhang, Y.; Shi, Q.; Liu, Y.; Wang, Y.; Meng, Z.; Xiao, C.; Deng, K.; Rao, D.; Lu, R. Hexagonal Boron Nitride with Designed Nanopores as a High-Efficiency Membrane for Separating Gaseous Hydrogen from Methane. J. Phys. Chem. C 2015, 119, 19826-19831.

(13) Yuan, Z.; Govind Rajan, A.; Misra, R. P.; Drahushuk, L. W.; Agrawal, K. V.; Strano, M. S.; Blankschtein, D. Mechanism and Prediction of Gas Permeation through Sub-Nanometer Graphene Pores: Comparison of Theory and Simulation. ACS Nano 2017, 11, 7974-7987.

(14) Yuan, Z.; Misra, R. P.; Rajan, A. G.; Strano, M. S.; Blankschtein, D. Analytical Prediction of Gas Permeation through Graphene Nanopores of Varying Sizes: Understanding Transitions across Multiple Transport Regimes. ACS Nano 2019, 13, 11809-11824.

(15) Zhao, J.; He, G.; Huang, S.; Villalobos, L. F.; Dakhchoune, M.; Bassas, H.; Agrawal, K. V. Etching Gas-Sieving Nanopores in SingleLayer Graphene with an Angstrom Precision for High-Performance Gas Mixture Separation. Sci. Adv. 2019, 5, No. eaav1851.

(16) He, G.; Huang, S.; Villalobos, L. F.; Vahdat, M. T.; Guiver, M. D.; Zhao, J.; Lee, W. C.; Mensi, M.; Agrawal, K. V. Synergistic $\mathrm{CO}_{2-}$ Sieving from Polymer with Intrinsic Microporosity Masking Nanoporous Single-Layer Graphene. Adv. Funct. Mater. 2020, 30, 2003979.

(17) Huang, S.; Li, S.; Villalobos, L. F.; Dakhchoune, M.; Micari, M.; Babu, D. J.; Vahdat, M. T.; Mensi, M.; Oveisi, E.; Agrawal, K. V. Millisecond Lattice Gasification for High-Density CO2- and O2Sieving Nanopores in Single-Layer Graphene. Sci. Adv. 2021, 7, No. eabf0116.

(18) Mahmood, J.; Lee, E. K.; Jung, M.; Shin, D.; Jeon, I.-Y.; Jung, S.-M.; Choi, H.-J.; Seo, J.-M.; Bae, S.-Y.; Sohn, S.-D.; et al. Nitrogenated Holey Two-Dimensional Structures. Nat. Commun. 2015, 6, 6486.

(19) Talukdar, M.; Behera, S. K.; Deb, P. Graphitic Carbon Nitride Decorated with $\mathrm{FeNi}_{3}$ Nanoparticles for Flexible Planar Micro-
Supercapacitor with Ultrahigh Energy Density and Quantum Storage Capacity. Dalton Trans. 2019, 48, 12137-12146.

(20) Talukdar, M.; Behera, S. K.; Bhattacharya, K.; Deb, P. Surface Modified Mesoporous G- $\mathrm{C}_{3} \mathrm{~N}_{4} @ \mathrm{FeNi}_{3}$ as Prompt and Proficient Magnetic Adsorbent for Crude Oil Recovery. Appl. Surf. Sci. 2019, 473, 275-281.

(21) Wang, Y.; Gao, B.; Yue, Q.; Wang, Z. Graphitic Carbon Nitride $\left(\mathrm{g}-\mathrm{C}_{3} \mathrm{~N}_{4}\right)$-Based Membranes for Advanced Separation. J. Mater. Chem. A 2020, 8, 19133-19155.

(22) Zhu, L.; Xue, Q.; Li, X.; Wu, T.; Jin, Y.; Xing, W. C2N An Excellent Two-Dimensional Monolayer Membrane for He Separation. J. Mater. Chem. A 2015, 3, 21351-21356.

(23) Xu, B.; Xiang, H.; Wei, Q.; Liu, J. Q.; Xia, Y. D.; Yin, J.; Liu, Z. G. Two-Dimensional Graphene-like $\mathrm{C}_{2} \mathrm{~N}$ : An Experimentally Available Porous Membrane for Hydrogen Purification. Phys. Chem. Chem. Phys. 2015, 17, 15115-15118.

(24) Deng, S.; Hu, H.; Zhuang, G.; Zhong, X.; Wang, J. A StrainControlled $\mathrm{C}_{2} \mathrm{~N}$ Monolayer Membrane for Gas Separation in PEMFC Application. Appl. Surf. Sci. 2018, 441, 408-414.

(25) Dion, M.; Rydberg, H.; Schröder, E.; Langreth, D. C.; Lundqvist, B. I. Van der Waals Density Functional for General Geometries. Phys. Rev. Lett. 2004, 92, 246401.

(26) Grimme, S. Accurate Description of van der Waals Complexes by Density Functional Theory Including Empirical Corrections. J. Comput. Chem. 2004, 25, 1463-1473.

(27) Grimme, S. Semiempirical GGA-Type Density Functional Constructed with a Long-Range Dispersion Correction. J. Comput. Chem. 2006, 27, 1787-1799.

(28) Qin, G.-q.; Du, A.-j.; Sun, Q. Charge- and Electric-FieldControlled Switchable Carbon Dioxide Capture and Gas Separation on a $\mathrm{C}_{2} \mathrm{~N}$ Monolayer. Energy Technol. 2018, 6, 205-212.

(29) Lee, K.; Murray, É. D.; Kong, L.; Lundqvist, B. I.; Langreth, D. C. Higher-Accuracy van der Waals Density Functional. Phys. Rev. B: Condens. Matter Mater. Phys. 2010, 82, 081101.

(30) Guan, Z.; Lian, C.-S.; Hu, S.; Ni, S.; Li, J.; Duan, W. Tunable Structural, Electronic, and Optical Properties of Layered TwoDimensional $\mathrm{C}_{2} \mathrm{~N}$ and $\mathrm{MoS}_{2}$ van der Waals Heterostructure as Photovoltaic Material. J. Phys. Chem. C 2017, 121, 3654-3660.

(31) Bai, Y.; Zhang, Q.; Xu, N.; Deng, K.; Kan, E. Efficient Carrier Separation and Band Structure Tuning of Two-Dimensional $\mathrm{C}_{2} \mathrm{~N} /$ GaTe van der Waals Heterostructure. J. Phys. Chem. C 2018, 122, 15892-15902.

(32) Giannozzi, P.; Baroni, S.; Bonini, N.; Calandra, M.; Car, R.; Cavazzoni, C.; Ceresoli, D.; Chiarotti, G. L.; Cococcioni, M.; Dabo, I.; et al. QUANTUM ESPRESSO: A Modular and Open-Source Software Project for Quantum Simulations of Materials. J. Phys. Condens. Matter 2009, 21, 395502.

(33) Giannozzi, P.; Andreussi, O.; Brumme, T.; Bunau, O.; Buongiorno-Nardelli, M.; Calandra, M.; Car, R.; Cavazzoni, C.; Ceresoli, D.; Cococcioni, M.; et al. Advanced Capabilities for Materials Modelling with Quantum ESPRESSO. J. Phys. Condens. Matter 2017, 29, 465901.

(34) Lejaeghere, K.; Bihlmayer, G.; Bjorkman, T.; Blaha, P.; Blugel, S.; Blum, V.; Caliste, D.; Castelli, I. E.; Clark, S. J.; Dal Corso, A.; et al. Reproducibility in Density Functional Theory Calculations of Solids. Science 2016, 351, aad3000.

(35) Prandini, G.; Marrazzo, A.; Castelli, I. E.; Mounet, N.; Marzari, N. Precision and Efficiency in Solid-State Pseudopotential Calculations. npj Comput. Mater. 2018, 4, 72.

(36) Grimme, S.; Antony, J.; Ehrlich, S.; Krieg, H. A Consistent and Accurate Ab Initio Parametrization of Density Functional Dispersion Correction (DFT-D) for the 94 Elements H-Pu. J. Chem. Phys. 2010, $132,154104$.

(37) Vydrov, O. A.; Van Voorhis, T. Nonlocal van der Waals Density Functional: The Simpler the Better. J. Chem. Phys. 2010, 133, 244103.

(38) Sabatini, R.; Gorni, T.; de Gironcoli, S. Nonlocal van der Waals Density Functional Made Simple and Efficient. Phys. Rev. B: Condens. Matter Mater. Phys. 2013, 87, 041108. 
(39) Tkatchenko, A.; Scheffler, M. Accurate Molecular van der Waals Interactions from Ground-State Electron Density and FreeAtom Reference Data. Phys. Rev. Lett. 2009, 102, 073005.

(40) van Setten, M. J.; Giantomassi, M.; Bousquet, E.; Verstraete, M. J.; Hamann, D. R.; Gonze, X.; Rignanese, G.-M. The PseudoDojo: Training and Grading a 85 Element Optimized Norm-Conserving Pseudopotential Table. Comput. Phys. Commun. 2018, 226, 39-54.

(41) Langreth, D. C.; Perdew, J. P. The Exchange-Correlation Energy of a Metallic Surface. Solid State Commun. 1975, 17, 14251429.

(42) Langreth, D. C.; Perdew, J. P. Exchange-Correlation Energy of a Metallic Surface: Wave-Vector Analysis. Phys. Rev. B: Solid State 1977, 15, 2884-2901.

(43) Dobson, J. F.; Gould, T. Calculation of Dispersion Energies. J. Phys. Condens. Matter 2012, 24, 073201.

(44) Ren, X.; Rinke, P.; Scheffler, M. Exploring the Random Phase Approximation: Application to CO Adsorbed on $\mathrm{Cu}(111)$. Phys. Rev. B: Condens. Matter Mater. Phys. 2009, 80, 045402.

(45) Colonna, N.; Hellgren, M.; de Gironcoli, S. Molecular Bonding with the RPAx: From Weak Dispersion Forces to Strong Correlation. Phys. Rev. B 2016, 93, 195108.

(46) Lu, D.; Li, Y.; Rocca, D.; Galli, G. Ab Initio Calculation of van der Waals Bonded Molecular Crystals. Phys. Rev. Lett. 2009, 102, 206411.

(47) Nguyen, H.-V.; de Gironcoli, S. Efficient Calculation of Exact Exchange and RPA Correlation Energies in the Adiabatic-Connection Fluctuation-Dissipation Theory. Phys. Rev. B: Condens. Matter Mater. Phys. 2009, 79, 205114.

(48) Kurth, S.; Perdew, J. P. Density-Functional Correction of Random-Phase-Approximation Correlation with Results for Jellium Surface Energies. Phys. Rev. B: Condens. Matter Mater. Phys. 1999, 59, 10461-10468.

(49) Ruzsinszky, A.; Perdew, J. P.; Csonka, G. I. A Simple but Fully Nonlocal Correction to the Random Phase Approximation. J. Chem. Phys. 2011, 134, 114110.

(50) Olsen, T.; Thygesen, K. S. Beyond the Random Phase Approximation: Improved Description of Short-Range Correlation by a Renormalized Adiabatic Local Density Approximation. Phys. Rev. B: Condens. Matter Mater. Phys. 2013, 88, 115131.

(51) Olsen, T.; Thygesen, K. S. Accurate Ground-State Energies of Solids and Molecules from Time-Dependent Density-Functional Theory. Phys. Rev. Lett. 2014, 112, 203001.

(52) Görling, A. Exact Exchange Kernel for Time-dependent Density-functional Theory. Int. J. Quantum Chem. 1998, 69, 265-277.

(53) Hellgren, M.; von Barth, U. Exact-Exchange Kernel of TimeDependent Density Functional Theory: Frequency Dependence and Photoabsorption Spectra of Atoms. J. Chem. Phys. 2009, 131, 044110.

(54) Colonna, N.; Hellgren, M.; de Gironcoli, S. Correlation Energy within Exact-Exchange Adiabatic Connection Fluctuation-Dissipation Theory: Systematic Development and Simple Approximations. Phys. Rev. B: Condens. Matter Mater. Phys. 2014, 90, 125150.

(55) Freeman, D. L. Coupled-Cluster Expansion Applied to the Electron Gas: Inclusion of Ring and Exchange Effects. Phys. Rev. B: Solid State 1977, 15, 5512-5521.

(56) Grüneis, A.; Marsman, M.; Harl, J.; Schimka, L.; Kresse, G. Making the Random Phase Approximation to Electronic Correlation Accurate. J. Chem. Phys. 2009, 131, 154115.

(57) Hellgren, M.; Colonna, N.; de Gironcoli, S. Beyond the Random Phase Approximation with a Local Exchange Vertex. Phys. Rev. B 2018, 98, 045117.

(58) Ren, X.; Tkatchenko, A.; Rinke, P.; Scheffler, M. Beyond the Random-Phase Approximation for the Electron Correlation Energy: The Importance of Single Excitations. Phys. Rev. Lett. 2011, 106, 153003.

(59) Klimeš, J.; Kaltak, M.; Maggio, E.; Kresse, G. Singles Correlation Energy Contributions in Solids. J. Chem. Phys. 2015, 143, 102816.
(60) Janesko, B. G.; Henderson, T. M.; Scuseria, G. E. Long-RangeCorrected Hybrids Including Random Phase Approximation Correlation. J. Chem. Phys. 2009, 130, 081105.

(61) Toulouse, J.; Gerber, I. C.; Jansen, G.; Savin, A.; Angyán, J. G. Adiabatic-Connection Fluctuation-Dissipation Density-Functional Theory Based on Range Separation. Phys. Rev. Lett. 2009, 102, 096404.

(62) Tkatchenko, A.; DiStasio, R. A.; Car, R.; Scheffler, M. Accurate and Efficient Method for Many-Body van der Waals Interactions. Phys. Rev. Lett. 2012, 108, 236402.

(63) Stöhr, M.; Sadhukhan, M.; Al-Hamdani, Y. S.; Hermann, J.; Tkatchenko, A. Coulomb Interactions between Dipolar Quantum Fluctuations in van der Waals Bound Molecules and Materials. Nat. Commun. 2021, 12, 137.

(64) Wang, Y.; Yang, Q.; Zhong, C.; Li, J. Graphene-like Poly(Triazine Imide) as $\mathrm{N}_{2}$-Selective Ultrathin Membrane for Postcombustion $\mathrm{CO}_{2}$ Capture. J. Phys. Chem. C 2016, 120, 2878228788.

(65) Li, Y.; Linghu, Y.; Wu, C. Separation Properties of Porous $\mathrm{MoS}_{2}$ Membranes Decorated with Small Molecules. ACS Appl. Mater. Interfaces 2020, 12, 20096-20102.

(66) Auerbach, S. M. Theory and Simulation of Jump Dynamics, Diffusion and Phase Equilibrium in Nanopores. Int. Rev. Phys. Chem. 2000, 19, 155-198.

(67) Blankenburg, S.; Bieri, M.; Fasel, R.; Müllen, K.; Pignedoli, C. A.; Passerone, D. Nanoporous Materials Porous Graphene as an Atmospheric Nanofilter. Small 2010, 6, 2266-2271.

(68) Vahdat, M. T.; Campi, D.; Colonna, N.; Villalobos, L. F.; Marzari, N.; Agrawal, K. V. Efficient $\mathrm{Kr} / \mathrm{Xe}$ Separation from Triangular g- $\mathrm{C}_{3} \mathrm{~N}_{4}$ Nanopores, a Simulation Study. J. Mater. Chem. A 2020, 8, 17747-17755.

(69) Perry, J. D.; Nagai, K.; Koros, W. J. Polymer Membranes for Hydrogen Separations. MRS Bull. 2006, 31, 745-749.

(70) Rohlfing, M.; Bredow, T. Binding Energy of Adsorbates on a Noble-Metal Surface: Exchange and Correlation Effects. Phys. Rev. Lett. 2008, 101, 266106.

(71) Ren, X.; Rinke, P.; Joas, C.; Scheffler, M. Random-Phase Approximation and Its Applications in Computational Chemistry and Materials Science. J. Mater. Sci. 2012, 47, 7447-7471.

(72) Rocca, D. Random-Phase Approximation Correlation Energies from Lanczos Chains and an Optimal Basis Set: Theory and Applications to the Benzene Dimer. J. Chem. Phys. 2014, 140, 18 A501.

(73) Cai, X.; Xie, W. J.; Yang, Y.; Long, Z.; Zhang, J.; Qiao, Z.; Yang, L.; Gao, Y. Q. Structure of Water Confined between Two Parallel Graphene Plates. J. Chem. Phys. 2019, 150, 124703.

(74) Stöhr, M.; Van Voorhis, T.; Tkatchenko, A. Theory and Practice of Modeling van der Waals Interactions in ElectronicStructure Calculations. Chem. Soc. Rev. 2019, 48, 4118-4154.

(75) Hermann, J.; DiStasio, R. A.; Tkatchenko, A. First-Principles Models for van der Waals Interactions in Molecules and Materials: Concepts, Theory, and Applications. Chem. Rev. 2017, 117, 47144758. 